\title{
Suicide ideation, depression and HIV among pregnant women in rural South Africa
}

\author{
Tamsen J. Rochat ${ }^{1,2^{\star}}$, Ruth M. Bland ${ }^{3}$, Mark Tomlinson ${ }^{2}$, Alan Stein $^{4}$ \\ ${ }^{1}$ Africa Centre for Health and Population Studies, University of KwaZulu-Natal, Mtubatuba, South Africa; \\ *Corresponding Author: trochat $@$ africacentre.ac.za \\ ${ }^{2}$ Department of Psychology, Stellenbosch University, Stellenbosch, South Africa \\ ${ }^{3}$ School of Medicine, University of Glasgow, Glasgow, UK \\ ${ }^{4}$ Section of Child and Adolescent Psychiatry, Department of Psychiatry, University of Oxford, Oxford, UK
}

Received 21 January 2013; revised 28 February 2013; accepted 8 March 2013

\section{ABSTRACT}

Background: Despite public perception to the contrary, pregnancy does not offer a protective effect for suicide ideation. In low and middle income countries (LMIC) multiple concurrent risk factors including unplanned pregnancies, testing for HIV, intimate partner violence and depression may increase suicide risk among pregnant women. We examine suicide ideation among women attending routine antenatal care in rural South Africa. Method: A consecutive series of 109 women in the second half of pregnancy were enrolled in a cross-sectional study at a large primary health care facility in a rural area of KwaZulu-Natal with high HIV prevalence. Using a mixed-methods approach, women were assessed using the Major Depression Section of the Structured Clinical Interview for Depression (SCID) for DSM-IV diagnosis; qualitative interview data were collected on women's experiences of suicide ideation and plans. Women were assessed two weeks after routine HIV testing as part of Prevention of Mother-to-Child Transmission (PMTCT) screening. Results: Both HIV-positive (49/109) and HIV-negative (60/109) women were assessed. Depression was high (51/109, 46.7\%) and equally common amongst HIV-positive and negative women. Suicide ideation was high $(30 / 109,27.5 \%)$ with thoughts of self harm closely linked to suicide plans. Age, previous history of depression and current depression were significantly associated with suicide ideation. Close to equal numbers of HIVpositive and negative women were suicidal. Suicide methods were frequently violent and most women cited family and partnership conflict related to an unplanned pregnancy or their newly
HIV-positive status as triggers to their suicide ideation. Conclusions: Rates of suicide ideation among pregnant women testing for HIV are high. Testing HIV-positive causes considerable distress, and among HIV-negative women unplanned and unwanted pregnancies are important risk factors for suicide ideation. Pregnancy brings women into increased contact with the health services with important opportunities for prevention and intervention.

Keywords: Suicide Ideation; Pregnancy; Depression; HIV; Rural; South Africa

\section{INTRODUCTION}

A recent review of global literature [1] found that pregnancy did not offer a protective effect for suicide ideation, with between $5 \%$ and $14 \%$ of women reporting suicide ideation during pregnancy or the postnatal period. Although suicide ideation and attempts are reported to be lower during pregnancy and the postpartum period compared to the general population of women, suicides account for up to $20 \%$ of postpartum deaths. This review also found that the presence of depression significantly increased the risk of death by suicide. Depression and psychosocial stressors are considered strong predictors of suicide ideation during pregnancy and the postnatal period [2]. Not only is suicide a leading cause of maternal death in the first year post-partum, but women tend to die violently by hanging or jumping as opposed to medication overdose, suggesting that the profile of women who complete suicide post-delivery is different to women in the general population [3]. Most research has been undertaken in the United States and Europe [4] finding prevalence ranging between $13 \%$ and $33 \%$ during pregnancy, and although there is a scarcity of research from low and middle income countries (LMICs), evidence of the pres- 
ence of suicide ideation during pregnancy in LMICs has emerged in the recent literature [4-8]. Research on antenatal depression in Bangladesh [6] found 14\% suicide ideation among pregnant women who had screened positive for probable depression on the Edinburgh Postnatal Depression Scale (EPDS). Reasons for suicide ideation included family and partner conflict, financial hardship and partner physical or psychological abuse. Similarly, in Pakistan [7], 11\% of all pregnant women interviewed reported suicide ideation in the last two weeks of pregnancy, of whom 67/148 (45\%) reported having attempted suicide. Recently, in an urban area of KwaZulu-Natal, South Africa [8], researchers screened women using the EPDS and found 38\% had probable depression and high numbers of women reported suicide ideation in the previous seven days $(145 / 380 ; 38.3 \%)$. In that study researchers found a significant association between probable depression and suicide ideation with risk factors including: being single and without support, being HIVpositive, having an unplanned pregnancy and a past history of depression. The link between antenatal depression and suicide ideation is important since in many Southern African settings the burden of antenatal depression is between $30 \%-47 \%$ [8-11] and risks are compounded by multiple factors including: HIV, with up to $43 \%$ of pregnant women in the worst affected areas testing HIV-positive [12], high rates of unplanned pregnancies [13] and intimate partner violence [14,15]. Suicide ideation among pregnant women in LMICs represents an important global mental health concern, in particular in HIV epidemic settings. The primary aim of this research is to examine the rates of suicide ideation among women attending routine antenatal care and testing for HIV during pregnancy, and to explore the relationships between suicide ideation, depression and HIV status. A secondary aim is to examine, qualitatively, women's subjective reporting of the factors instrumental to suicide ideation.

\section{METHODS}

\subsection{Research Context}

The research was undertaken in a predominantly rural area with high HIV prevalence in South Africa [16]. The Hlabisa sub-district health services include a local district hospital with 250 beds and 17 decentralized primary health care clinics serving a population of approximately 220,000 . This study was undertaken at the largest periurban primary health care clinic in the sub-district, staffed by 20 - 30 nurses and offering a full range of primary health care services to over 10,000 patients per month including a large antenatal and postnatal outpatient service, an average of 160 first time antenatal attendees per month and between 70 - 100 normal deliver- ies per month.

\subsection{Recruitment Procedure}

This nested study recruited a sub-sample of women from a larger cohort of baseline participants enrolled at three clinics in the sub-district [17]. Women were invited to complete an in-depth assessment interview which took place 3 to 4 weeks after baseline, which is described in detail elsewhere [11]. To be eligible, women were required to be attending routine antenatal care, at least 16 years of age, in the second half of pregnancy, and living in the study area. Women with severe or chronic health problems such as diabetes or known HIV-related illness were referred for specialist services and excluded from this research. All women were required to have tested for HIV as part of PMTCT and have received their HIV results at baseline. Informed consent was obtained in writing and the study received ethical approval from the Biomedical Ethics Review Board of the University of KwaZulu-Natal and the Oxford Tropical Research Ethics Committee (OXTREC).

\subsection{Sample}

Sample recruitment is illustrated in Figure 1. Of 156 eligible baseline participants, $112(72 \%)$ completed the second assessment and $44(28 \%)$ were lost to follow up. Three women were removed from data analysis due to missing data.

\subsection{Depression Assessment}

Pregnant women were interviewed using the Major Depression Section of the Structured Clinical Interview for DSM-IV diagnoses [18]. A detailed description of the depression assessment method and scoring, and the process of translation and cultural validation has been published [11]. A mixed-method approach was used with qualitative data recorded for each symptom, including suicide ideation, in order to examine personal and cultural experiences of depressive symptoms. This mixedmethod approach captures both the presence/absence of suicide ideation according to a standard definition, but also explores contextual factors that inform the experience of depression and suicide ideation. Women also completed the 10-item Edinburgh Postnatal Depression Scale (EPDS), the most widely validated screening tool for depression during pregnancy [19].

\subsection{Suicide Ideation Assessment}

Suicide ideation was examined and recorded using the SCID question guides including: Have you been thinking you would be better off dead? Have you been thinking about hurting yourself? Have you thought about or 


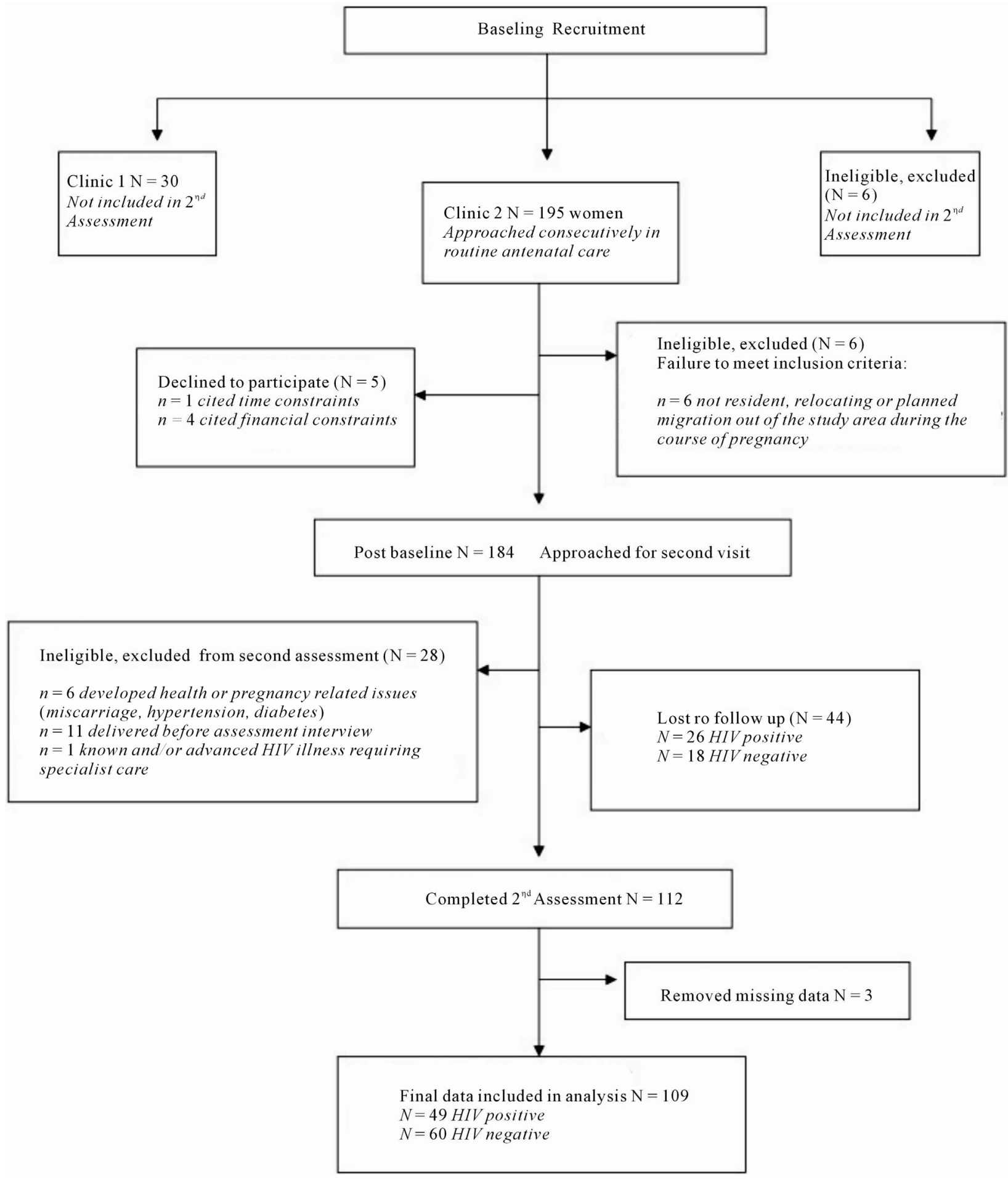

Figure 1. Recruitment summary [11].

made plans about how you may hurt yourself? Have you ever attempted to harm yourself?

Further qualitative probes for women reporting suicide ideation included: What has this been like for you? What do you think is the main reason you have wanted to end your life? At the times when you are thinking of ending your life what are the things that are bothering you the most? All women reporting suicide ideation were referred to a clinical psychologist for further management.

\subsection{Data Analysis}

The coding and scoring of the depression data has 
been described [11] resulting in a dichotomous $0 / 1$ score depending on symptom presence/absence, severity and duration. An overall depression (0/1) outcome was determined using the DSM diagnostic criteria to evaluate symptoms by participant. Data were double entered for accuracy into STATA11 software for data analysis. Principal component analysis techniques were used to examine groups or clusters of symptom variables around a main or principal component, and to examine the spread of symptom variance within that component [11]. Median age t-test and Chi Square tests were used to test differences between sample characteristics of suicidal and non-suicidal women. Logistical regression was used to examine associations between depression, HIV, and suicide ideation. Sensitivity of the EPDS screening item for suicide ideation was tested using the Vassar Clinical Calculator (www.vassarstats.net). Content analysis of qualitative interview notes were used to identify categories of responses for suicide ideation. Once common categories were identified and coded these were assessed using a constant comparative analysis technique to determine summary concepts within each category which were inclusive and mutually exclusive. Categories of coded data were entered, organized and analyzed with NVivio software to establish counts and frequencies.

\section{RESULTS}

\subsection{Sample Characteristics}

The sample characteristics of the 109 women assessed are reported in Table 1. While a third more HIV-positive women were lost to follow up than HIV-negative women, an examination of data available from baseline did not find significant differences between women lost to follow up and those interviewed [11]. The overall sociodemographic characteristics of this sample were similar to those reported for antenatal attendees in the baseline study [17]. Most women in the sample were young, had completed some or all of their secondary education, and were from low income groups. Most were unmarried, but in a stable relationship with the father of the child, and most were living with their families rather than cohabiting with partners. Reproductive health data showed that high numbers of women tested HIV-positive (49/109; $44.9 \%$ ) and most women $(85.3 \%)$ had an unplanned pregnancy. No significant association was found between unplanned pregnancies and depression, or HIV and depression in univariate analysis, although a trend is noted between HIV and antenatal depression (OR1.84 [0.86 3.95] $\mathrm{p}=0.117$ ).

As shown in Table 1 suicidal women tended to be slightly older, with a median age of 25 years compared to a median of 22 years for non-suicidal women. Median
Table 1. Sample characteristics of suicidal and non suicidal pregnant women.

\begin{tabular}{|c|c|c|c|}
\hline \multirow[t]{2}{*}{ Characteristics } & $\begin{array}{c}\text { Not suicidal } \\
\quad \mathrm{N}=79\end{array}$ & $\begin{array}{c}\text { Suicidal } \\
\mathbf{N}=\mathbf{3 0}\end{array}$ & Difference \\
\hline & n (\%) & n (\%) & $\mathbf{p}^{*}$ \\
\hline $\begin{array}{l}\text { Age } \\
\text { Median } \\
\text { Range }\end{array}$ & $\begin{array}{c}22 \\
16-37\end{array}$ & $\begin{array}{c}25 \\
17-39\end{array}$ & $p=0.047$ \\
\hline Education & & & $\mathrm{p}=0.695$ \\
\hline No education & $12(15 \%)$ & $2(7 \%)$ & \\
\hline Completed Primary & $26(33 \%)$ & $12(40 \%)$ & \\
\hline Some secondary & $22(28 \%)$ & $10(33 \%)$ & \\
\hline Completed secondary & $19(24 \%)$ & $6(20 \%)$ & \\
\hline Marital status & & & $\mathrm{p}=0.235$ \\
\hline Unmarried & $74(94 \%)$ & $26(87 \%)$ & \\
\hline Married & $5(6 \%)$ & $4(13 \%)$ & \\
\hline In stable relationship & & & $\mathrm{p}=0.587$ \\
\hline Yes & $72(91 \%)$ & $26(87 \%)$ & \\
\hline No & $5(6 \%)$ & $2(7 \%)$ & \\
\hline Missing & $2(3 \%)$ & $2(7 \%)$ & \\
\hline Cohabiting with father & & & $\mathrm{p}=0.203$ \\
\hline Yes & $12(15 \%)$ & $9(30 \%)$ & \\
\hline No & $45(57 \%)$ & $15(50 \%)$ & \\
\hline Missing & $22(28 \%)$ & $6(20 \%)$ & \\
\hline Children with father & & & $\mathrm{p}=0.893$ \\
\hline First child & $41(52 \%)$ & $10(33 \%)$ & \\
\hline At least one other & $38(48 \%)$ & $20(67 \%)$ & \\
\hline Living arrangements & & & $\mathrm{p}=0.570$ \\
\hline Family & $65(82 \%)$ & $22(73 \%)$ & \\
\hline Non Family & $11(14 \%)$ & $6(20 \%)$ & \\
\hline Missing & $3(4 \%)$ & $2(7 \%)$ & \\
\hline Regular income & & & $\mathrm{p}=0.424$ \\
\hline Yes & $41(52 \%)$ & $13(43 \%)$ & \\
\hline No & $38(48 \%)$ & $17(57 \%)$ & \\
\hline Grant assistance & & & $\mathrm{p}=0.092$ \\
\hline Child Support Grant & $36(46 \%)$ & $16(53 \%)$ & \\
\hline Care Dependency Grant & $0(0 \%)$ & $1(3 \%)$ & \\
\hline No grant & $43(54 \%)$ & $12(40 \%)$ & \\
\hline Missing & $0(0 \%)$ & $1(3 \%)$ & \\
\hline HIV status & & & $\mathrm{p}=0.514$ \\
\hline HIV-positive & $34(43 \%)$ & $15(50 \%)$ & \\
\hline HIV-negative & $45(57 \%)$ & $15(50 \%)$ & \\
\hline Pregnancy & & & $\mathrm{p}=0.634$ \\
\hline Planned & $27(29 \%)$ & $3(20 \%)$ & \\
\hline Unplanned & $66(71 \%)$ & $12(80 \%)$ & \\
\hline
\end{tabular}

${ }^{*} \mathrm{p} \geq 0.50$ significance level. 
age t-test found this difference to be significant ( $\mathrm{p}=$ 0.47); logistic regression found that with each increasing year of age women were $8 \%$ more likely to be suicidal (OR $1.08[0.9-1.1] \mathrm{p}=0.056)$ at borderline significance level. No other significant differences or associations with sample characteristics and suicide ideation were found.

\subsection{Suicide Ideation}

Thirty of the 109 women $(27.5 \%$ CI 19 - 36) scored positive for suicide ideation on the SCID, almost all 27/ $30(90 \%)$ of these were diagnosed with depression.

\subsection{Prevalence of Depression}

Prevalence of depression was high $(51 / 109 ; 47 \%)$ and tended to be chronic, with over half of the depressed women 34/51 (67\%) reporting episode duration greater than two months. Over half of all depressed women 27/51 (53\%) were suicidal; three women reporting suicide ideation were not depressed. As shown in Table 2, depression was significantly associated with reporting suicide ideation in both univariate and multivariate analysis, with depressed women being 20 times more likely to be suicidal than non-depressed women (AOR21.9 [5.8 - 82] $p=0.000$ ). Given the small sample size and wide confidence intervals, in a larger sample one would expect a larger effect size. Principle component analysis (PCA) of depression symptoms [11] extracted one component (eigenvalue $=4.80176$ ) which accounted for most of the variance (proportion $=0.2668$ ). Suicidal thoughts had the highest factor loading $(0.3280)$ followed closely by suicidal plans $(0.2937)$, depressed $\operatorname{mood}(0.2915)$ and loss of interest (0.2963).

\subsection{Previous Episode of Depression}

Of all the women assessed, only a small number of women $(15 / 109 ; 14 \%)$ reported any prior history of depression. Of the 51 depressed women: 8/51 reported a prior episode of depression which had resolved before the current pregnancy. Of these $2 / 8$ reported a prior episode of depression but were not currently suicidal while $6 / 8$ reported a prior episode of depression, had current depression and current suicide ideation. Of the 58 nondepressed women: 7/58 reported a prior episode of depression of whom $6 / 7$ had a previous episode of depression but were not currently depressed or suicidal, while $1 / 7$ had a prior episode of depression, no current depression, but was currently suicidal.

While a previous episode of depression was not associated with a current depression (OR 1.35 [0.4 - 4.0] p = $0.585)$, a previous history of depression was associated with being currently suicidal in both univariate (OR 2.7 $[0-8-8.2] \mathrm{p}=0.082$ ) and multivariate analysis (AOR $3.4[0.8-14] \mathrm{p}=0.097)$ making women close to three times more likely to be suicidal, at borderline significance (see Table 2).

\subsection{EPDS Suicidal Screening Item}

Item 10 on the EPDS "the thought of harming myself has occurred to me" was found to have good sensitivity of $77 \%$ (CI 57 - 89) and excellent specificity of $92 \%$ (CI 83 - 96) for detecting suicide ideation; the positive likelyhood ratio $(\mathrm{LR}+)$ weighted by prevalence showed that women scoring positive on this EPDS item were almost four times more likely to be suicidal based on the SCID (AOR 3.83 [1.8 - 8]). Scoring positive on this item was significant regardless of whether women reported frequency of "thoughts of self-harm" to be: hardly ever ( $p=$ $0.010)$ sometimes $(p=0.000)$ or quite often $(p=0.000)$ on EPDS Item 10.

\subsection{HIV Status}

HIV test results for the women enrolled in this study indicated that high numbers of women 49/109 (44.9\%) were HIV-positive. Equal numbers of HIV-positive women were suicidal $(\mathrm{n}=15 / 30)$ when compared to HIVnegative women $(n=15 / 30)$. Of the three women who were not depressed, but were suicidal, 2/3 were HIVpositive.

Table 2. Relationship between depression and suicide status.

\begin{tabular}{cccccc}
\hline NS & S & Chi Square & Univariate Logistic & Multivariate Logistic \\
\hline Depression & 55 & 3 & $\mathrm{p}=0.000$ & OR 20.6 (5- 74) $\mathrm{p}=0.000$ & AOR 21.9 (5.8 -82$) \mathrm{p}=0.000$ \\
Yes & 24 & 27 & & & \\
No & & & $\mathrm{p}=0.074$ & OR 2.7 $(0-8-8.2) \mathrm{p}=0.082$ & AOR $3.4(0.8-14) \mathrm{p}=0.097$ \\
Previous Depression & 8 & 7 & & & \\
Yes & 71 & 23 & & & \\
No & & &
\end{tabular}




\subsection{Suicidal Plans and Means}

Of 30 suicidal women, 24 (80\%) reported having made plans towards self harm with slightly more HIVpositive women $(n=13 / 24)$ reporting that they had made plans compared to HIV-negative women $(n=11 / 24)$. Self harm means included: drinking paraffin or poison $(13 / 24)$; drowning themselves (5/24); hanging (3/24); setting themselves alight $(2 / 24)$; or throwing themselves in front of a car (1/24). The three women reporting suicide ideation who were not currently depressed, also reported making suicide plans.

\subsection{Suicidal Attempts}

Only two women reported having made previous suicide attempts, both of whom were HIV-positive. Despite high reporting of suicide ideation during follow up referrals, in the majority of cases (21/30) women were judged by a clinical psychologist not to need in-patient hospital care, but instead received outpatient support and counseling, and home-based follow up with support from family networks and social workers.

\subsection{Qualitative Data}

Content analysis found that women reporting suicide ideation expressed their suicidal ideation with a similar pattern of narratives, favouring "better off dead" over more directly stating they would like "to commit suicide or harm themselves". Death was frequently described as a reprieve from what appeared to be insurmountable, acute or chronic problem situations. In response to qualitative probes around the triggers for their suicide ideation, most women $(25 / 30$ or $83 \%)$ reported partnership conflict being closely linked to suicide ideation:

"Especially if I have quarreled with my partner, then these feelings are worse" (P19)

"In my lowest times, when I want to end it all I am thinking about my partner, that is the matter that is concerning me the most when I feel this way" (P38)

Having an unplanned pregnancy or having tested HIVpositive were reported as instrumental to both the partnership conflict and their suicidal feelings:

"Often I think about the love that is lost between me and my partner over this pregnancy and then I just feel like I want to be dead, all is lost now and I don't have a reason to live, he does not want me and he does not want the baby" (P31)

"This unplanned pregnancy and that I am not having any hope in my life, in the future or with my partner after I got my blood results, I thought I could be a great wife before, but things aren't the same anymore, so there have been times that I have thought about just going ahead and ending my life" (P73)
The five women who did not report partnership conflict reported family conflict resulting from the unplanned pregnancy as leading to the suicidal thoughts:

"Due to the situation that I face of having no parents and my sister having left her kids for me, and my uncle having so much anger that I am pregnant, I just feel like screaming a lot, most of the time my thoughts become wild and I feel like disappearing and leaving everything behind, that I should be dead so it can all go away" (P40)

"I feel such pain in my heart, I have all these thoughts and there are no solutions, and my mother is just scolding me all the time, I think I will be better if I was just dead" (P57)

Particular events were cited as leading to a sense of crisis and increased suicidal feelings, two scenarios were most common in the content analysis: firstly, feeling overwhelmed by learning one's HIV-positive status among HIV-positive women; and secondly, feeling overwhelmed by the implications of the unwanted pregnancy in partnerships among both HIV-positive and negative women.

HIV-positive suicidal women made statements such as:

"I was not happy before, but now with my HIV status and the pregnancy it has made it worse, like because of that I just think of ending it all a lot of the time" (P12)

"The cause of all these worries I have is that I am HIV-positive, my life really changed that day, I haven't told anybody, I am scared, where to start...all of these really bad feelings started when I got my results, when I learnt that I was positive" (P36)

While one HIV-negative women stated:

"When I call him he always says he is busy, sometimes he sends me a please call me and when I call him he says whatever he likes... what this man is doing to me right now... maybe when I take the phone and buzz him and he calls and tells me all that he wants, abusing me... then I think about a lot of things when that happens, I think about killing myself, or even aborting the baby" (P43)

The one women who was not currently depressed, HIV-negative, but was suicidal, reported being in an acute crisis over the unplanned pregnancy, worsened by her young age, family conflict and partner abandonment, and currently feeling destitute, she stated:

"It has been difficult right from the beginning of this pregnancy, the situation at home with my uncle was not good, they were chasing me away and shouting at me saying I should go to where I got this baby from, that they don't want me (becomes tearful) they are very disappointed in me (tearful) the father (of this baby) granny has said she will try to help, but then he (referring to father of the baby) just left me like that, when I was four months pregnant, he just disappeared and I haven't seen 
him, he hasn't called or anything, it hurts a lot that my family is chasing me, I don't even know where I will go after the delivery, I don't have a home, my parents are passed so I had to stay with my uncle and I don't have a home now, I have lost everything, it's better to end my life things are so bad" (P1)

Despite high rates of suicide ideation, and the catastrophic consequences for both the mother and foetus, almost no women made reference to the implications of suicide for the current foetus or any of their other children, except in the case of one HIV-positive woman who stated:

"I am going to die anyway, not matter how you look at it, either I will leave this baby behind and somebody will abuse it, or worse it will die with much suffering because I have infected it, it is better if I just end it for both of us now" (P27).

\section{DISCUSSION}

Globally, suicide rates have increased by $60 \%$ in recent decades [20,21], with growing epidemics in Eastern Europe and Asia. In Africa suicide statistics are equally concerning, but reliable data across the region is scarce [22]. As compared to other Southern African countries, more suicide data are available in South Africa, where the national lifetime prevalence suggests that $9.1 \%$ of South Africans experience suicide ideation [23] and where significant drop-offs are seen from thoughts of suicide $(9.1 \%)$ to plans $(3.8 \%)$ and attempts $(2.9 \%)$. Epidemiological research on completed suicide suggests that South African rates are elevated, but that patterns of suicide are similar to other countries, in particular with males found to be at greater risk than females of fatal suicide [24]. Very little research has examined suicide ideation among pregnant women, but that which has reports significant risk [8].

\subsection{Rates of Suicide Ideation}

The KwaZulu-Natal province of South Africa, where this research took place, is heavily affected by poverty and HIV [25] and the rate of suicide ideation among the general population has been found to be as high as $25 \%$ in community based samples [26]. This research found equally high rates of suicide ideation (27\%) among pregnant women in a rural KwaZulu-Natal setting heavily affected by HIV using a clinical interview method. This suicide ideation rate is in line with recent epidemicological reviews from high income countries finding suicide ideation as high as $33 \%$ among pregnant women $[1,2,4]$, but it is substantially higher than those reported in Bangladesh and Pakistan [6,7] where screening methods detected rates ranging from $11 \%-14 \%$.

Likewise, depression in this sample (47\%) was also higher than the estimated antenatal prevalence of $22 \%$ in a meta-analysis of studies in LMIC settings [27], and higher than the rates of probable depression (between $18 \%-33 \%$ ) in the two above-mentioned studies examining suicide risk among pregnant women in Asia. While the rates of depression and suicide ideation in this sample are higher than most research from LMICs they are not discordant with other available evidence from South Africa. Rates of suicide ideation in this research were found to be lower than the $38 \%$ reported in research from an urban sample of pregnant women in KwaZulu-Natal [8]. It is possible that in this rural, more "culturally traditional" sample, women may have under-reported suicide ideation [28], but it is equally possible that the clinical interview method, with its inclusion of detailed data on severity, frequency and duration may offer a more accurate reflection of suicide rates than studies using screening methods.

\subsection{Correlates of Suicide Ideation}

Globally, depression is known to be associated with increased risk of suicide, or serious suicide attempts, with over $60 \%$ of all suicides occurring in persons with depression [4]. This research found suicide ideation was significantly associated with antenatal depression. This finding replicates findings reported in the literature on depression and suicide ideation in pregnant women from both high income [1,2] and LMIC settings [6-8]. This increasing evidence lends support to calls for increased public health investments to ensure adequate screening, prevention and treatment for pregnant and postpartum women in these LMIC [29-31]. Our findings on the sensitivity of the EPDS screening item for depression, which is similar to other research in South Africa [8], lends support to the notion that simple short screens may be effective in identifying suicide ideation among pregnant women.

Similar to previous research $[1,2]$ we found associations with both age and prior depressive episodes. Women who had a previous episode of depression were three times more likely to be at risk of current suicide ideation. Depression (either prior to or during the pregnancy) appears to be strongly associated with suicide ideation in pregnant women globally. While a prior episode of depression was not independently associated with antenatal depression (OR 1.35 CI $0.4-4.0 \mathrm{p}=0.585$ ) the numbers were too small for this to be conclusively examined in data analysis [11]. Interestingly, three suicidal women had no current or prior depression and one suicidal woman reported a prior depression but no current depression, raising the possibility, as has been postulated in some reports $[2,4]$, that for some women depression and suicide ideation may be independent phe- 
nomena. We found no significant association between unplanned pregnancies and suicide ideation, as has been shown in other research in pregnancy in South Africa $[8,9]$, however, rates of unplanned pregnancies were pervasive in this sample, and substantial qualitative data supports the notion that for suicidal women an unplanned pregnancy, together with either partner or family conflict over the unplanned nature of the pregnancy, was a significant psychosocial stressor which frequently triggered suicide ideation. Partner conflict and intimate partner violence are strongly associated with antenatal depression in this region $[9,15]$ and with both antenatal depression and suicide ideation in other LMICs $[6,7,27,32]$.

\subsection{Suicide Ideation, Depression and HIV}

Rates of suicide ideation among HIV-positive women in this research were high $15 / 49$ (31\%) and similar to research among non-pregnant adults testing for HIV in South Africa which found rates of suicide ideation between 17\% (72 hours post testing) and 24\% (6 weeks post testing) among those testing HIV-positive [33]. In high-income settings research shows that suicide ideation, plans and attempts are elevated among persons living with HIV [34-36]. In the United States, for example, at least a third of women living with HIV report attempting suicide, mostly in the first week or month after learning their diagnosis, and having children is a significant predictor of suicide attempts [37]. More recently, research in Europe demonstrated that access to HIV treatment may lower suicide in HIV-infected individuals [38,39], however, regardless of access to treatment, suicide ideation still remains over three-times higher amongst HIVpositive persons than in the general population [40,41]. While no significant association was found between HIV status and suicide ideation in this research, qualitative data showed that slightly more HIV-positive women expressed plans towards suicide than did HIV-negative women and the two women who had previously attempted suicide were HIV-positive. However, it is noteworthy that rates of suicide ideation among women testing HIV-negative were also high 15/60 (25\%). Among HIV-negative women, unplanned and unwanted pregnancies were important risk factors for suicide ideation. These results suggest that both HIV-positive and negative women are at high risk of both antenatal depression and suicide ideation, and as such screening may be better placed in primary health care or routine antenatal services, such as universal PMTCT screening and testing offered to all antenatal attendees rather than exclusively placed within HIV treatment programmes.

The relationship between HIV, antenatal depression and suicide ideation (regardless of whether one tests HIV-positive during pregnancy, or is HIV-positive prior to becoming pregnant) is a complex one. In baseline research [13] using the EPDS prior to HIV testing we found no significant association between subsequent HIV status and EPDS probable depression. In this research, using a clinical interview method and assessing women 2 - 3 weeks post HIV testing, we find only evidence of a trend in association $(p=0.117)$. On the other hand, similar research using the EPDS in an urban area of KwaZulu-Natal did find a significant association between being HIV-positive, antenatal depression, and having suicidal thoughts [8]. That research differed from ours methodologically in that it used a screening measure as opposed to a clinical interview method, and lacked specificity around the timing and accuracy of HIV status. In our study, women were assessed 2 - 3 weeks post HIV testing, uptake of test results were part of inclusion and exclusion criteria, and self reported HIV status was independently verified against clinical records $[11,13]$ whilst in the urban study [8] participants' HIV status was collected retrospectively and by self report, with $19.3 \%$ of women reporting their HIV status to be currently "unknown". Further longitudinal research with larger community based samples and using clinical interview methods is required to better elucidate the relationship of HIV status, antenatal depression and suicide ideation in HIV epidemic settings.

In this research, significant qualitative data supports the notion that testing HIV positive adds significant psychosocial stress triggering suicidal ideation, if not depression, although we have previously demonstrated that the chronic nature of depression in this sample meant that the depression likely preceded HIV diagnosis [11]. Thus, suicide ideation may be a feature of a depressed woman's response to an HIV diagnosis during pregnancy, since depression is frequently associated with negative thinking, poor problem solving and poor health perceptions $[9,42]$.

\subsection{Suicide Plans and Means}

Suicide plans and preferred means are important factors in the development of suicide prevention strategies, in particular since what is referred to as "means reducetion" (reducing access to the preferred means of suicide) has been shown in systematic reviews to have success in reducing suicide [20]. In this research, suicidal plans for pregnant women followed suicide ideation closely, and the drop-off from ideation to suicide plans seen in the general population is less evident for pregnant women in our research [23]. High rates of suicide plans linked to ideation has been noted in other emerging literature on suicide in the perinatal period [4]. Means of suicide described in this research tended to be violent (poising, drowning, hanging) a finding also supported by a recent 
literature review [1]. In South Africa, hanging (36.2\%), shooting (35\%), and poisoning, (9.8\%) are the most common methods used in fatal suicide. However, these means are more commonly reported amongst men, who make up the majority of fatal suicides [43]. Overdosing on medication is more closely associated with attempted (non-fatal) suicides, which in turn is more frequently observed among women, particularly in high-income settings [20]. No women in our research study indicated a plan to use medication as a means to overdose.

Some research suggests that reported "means" of suicide become more severe and lethal as suicide intent increases, and that intent may be inferred by the degree of violence reported in suicide "means" [22]. However, literature also postulates that reports of plans and means may be affected by issues of access, and that fatalities, in particular in LMIC settings, may be complicated by a lack of access to health care and ambulatory services post suicide attempts. The high level of reporting of what the literature would describe as "increasingly lethal and violent means" such as hanging, drowning and poisoning in this research may indeed suggest that women had higher suicidal intent, but it may also simply reflect that women in rural areas, with lowered access to health care generally, and little access to lethal medications specifically, are left with fewer and more lethal options to consider when contemplating suicide, as has been shown in China, one of the few places where suicide rates among females exceed that of males [43].

\section{CONCLUSIONS}

Suicide is a significant and growing global mental concern [20]. Globally, there is growing concern around suicide ideation among pregnant and postnatal women. In this research rates of antenatal depression and suicide ideation were found to be concerningly high. Improvements in screening and preventative efforts for pregnant women are urgently needed. Primary health systems could take advantage of the increased contact with health services that results from pregnancy. The South African national suicide prevention strategy [22], like many evolving international suicide prevention strategies [21] suggests that whenever possible suicide prevention should be localized and culturally appropriate, and should target high risk groups [22]. However, few strategies currently target pregnant or postnatal women. This is in part pragmatic since fatal suicides remain more common amongst men, but are also driven by the limited understanding of the risks of suicide among pregnant and postnatal women, both among health care professionals and the general public.

Further research is required to examine the risk for suicide ideation in larger, community-based samples of pregnant women to determine population based prevalence and to guide the development of targeted prevention strategies and advocacy. In HIV epidemic settings, routine HIV testing in PMTCT programmes mean that many women learn their HIV-positive status for the first time during pregnancy [44-46]. It is well accepted that learning one's HIV-positive diagnosis is a negative life event, shown to be associated with onset of depression among non-pregnant women in South Africa [36,47], Africa [5], and internationally [36,48]. In this research HIV-negative women were also at high risk. It is plausible that testing HIV negative, but feeling unsure of one's capacity to prevent HIV infection in the future, due to low autonomy and relationship power $[14,15,49]$ may prove an equally stressful event for women. Cumulative overlapping risks factors around unplanned pregnancies, poverty, family conflict, a lack of partner support, intimate partner violence may trigger suicide risks for women [50-53] even in the absence of a current HIVpositive diagnosis. It is also plausible that efforts to screen for, prevent and treat antenatal depression and suicide risk may also strengthen HIV prevention efforts [46,54].

While international evidence suggests that health care education and screening may prevent suicides [20] the introduction of routine screening and prevention strategies in LMICs is not without its challenges. Efforts are hampered by shortages of health care professionals [55-57], overburdened clinics [58] and a lack of training and support for lay counsellors and community health care workers who work in time-pressured, task-heavy, roles where compassion fatigue and burnout is commonplace [58-60]. Since universal screening is seldom costeffective in the absence of access to treatment [61], significant policy and public health investments are required to make suicide prevention and intervention feasible for pregnant women in these settings. A lack of health care provider awareness and training has also been shown to account for much inequity in access to treatment for depression [62]. Similarly it is probable that depression itself may inhibit a women's engagement with health services during pregnancy and as such community based strategies must be considered [30,31].

The lack of epidemiological studies from LMICs [63] using clinical interview methods substantially limits the ability to advocate for appropriate investments of public health resources [64].

This research is strengthened by its use of clinical interview methods, and by being one of only a few studies in Africa to examine suicide ideation in pregnancy. However, findings should be considered preliminary given the relatively small sample size and risk of selection bias introduced by the numbers of women lost to follow up. Replication in larger community based samples is an 
urgent research priority.

While most of the findings of this research are concordant with other LMIC and international research, the high rates of depression and the equally high co-morbid HIV may reflect a particular risk profile or factors present in this population of women which may not generalisable to other antenatal populations with lower perinatal depression, or lower HIV infection.

\section{ACKNOWLEDGEMENTS}

We thank the women who participated in this study and the KwaZulu-Natal Department of Health. This study was funded by grants from University of Oxford (HQ5035), the Tuixen Foundation (9940), and the Welcome Trust (082384/Z/07/Z and071571). Tamsen Rochat receives salary support from the Wellcome Trust-funded Africa Centre, and data analysis was supported through a grant from the American Psychological Foundation. The statistical input of Mr James Ndirangu (Africa Centre) and epidemiological input of Professor Marie-Louise Newell (Africa Centre) are gratefully acknowledged.

\section{REFERENCES}

[1] Lindahl, V., Pearson, J.L. and Colpe, L. (2005) Prevalence of suicidality during pregnancy and the postpartum. Archives of Women's Mental Health, 8, 77-87. doi:10.1007/s00737-005-0080-1

[2] Gavin, A., et al. (2011) Prevalence and correlates of suicidal ideation during pregnancy. Archives of Women's Mental Health, 14, 239-246.

[3] Oates, M. (2003) Suicide: The leading cause of maternal death. The British Journal of Psychiatry, 183, 279-281. doi:10.1192/bjp.183.4.279

[4] Gentile, S. (2011) Suicidal mothers. Journal of Injury and Violence Research, 3, 90.

[5] Kinyanda, E., Hjelmeland, H. and Musisi, S. (2005) Negative life events associated with deliberate self-harm in an African population in Uganda. Crisis: The Journal of Crisis Intervention and Suicide Prevention, 26, 4-11. doi:10.1027/0227-5910.26.1.4

[6] Asad, N., et al. (2010) Prevalence of suicidal thoughts and attempts among pregnant Pakistani women. Acta Obstetricia et Gynecologica Scandinavica, 89, 1545-1551. doi:10.3109/00016349.2010.526185

[7] Gausia, K., et al. (2009) Antenatal depression and suicide ideation among rural Bangladeshi women: A community based study. Archives of Women's Mental Health, 12, 351358. doi:10.1007/s00737-009-0080-7

[8] Manikkam, L. and Burns, J.K. (2012) Antenatal depression and its risk factors: An urban prevalence study in KwaZulu-Natal. South African Medical Journal, 102, 940-944.

[9] Hartley, M., et al. (2011) Depressed mood in pregnancy: Prevalence and correlates in two Cape Town peri-urban settlements. Reproductive Health, 8, 9. doi:10.1186/1742-4755-8-9

[10] Sawyer, A., Ayers, S. and Smith, H. (2010) Pre- and post- natal psychological wellbeing in Africa: A systematic review. Journal of Affective Disorders, 123, 17-29. doi:10.1016/j.jad.2009.06.027

[11] Rochat, T.J., et al. (2011) The prevalence and clinical presentation of antenatal depression in rural South Africa. Journal of Affective Disorders, 135, 362-373. doi:10.1016/j.jad.2011.08.011

[12] Department of Health (2010) National Antenatal Sentinel HIV and Syphilis Prevalence Survey in South Africa, 2009. National Department of Health, Pretoria.

[13] Rochat, T.J., et al. (2006) Depression among pregnant rural South African women undergoing HIV testing. Journal of the American Medical Association, 295, 1376-1378. doi:10.1001/jama.295.12.1376

[14] Dunkle, K.L., et al. (2004) Gender-based violence, relationship power, and risk of HIV infection in women attending antenatal clinics in South Africa. Lancet, 363, 1415-1421. doi:10.1016/S0140-6736(04)16098-4

[15] Jewkes, R.K., et al. (2010) Intimate partner violence, relationship power inequity, and incidence of HIV infection in young women in South Africa: A cohort study. Lancet, 376, 41-48. doi:10.1016/S0140-6736(10)60548-X

[16] Tanser, F., et al. (2008) Cohort profile: Africa Centre Demographic Information System (ACDIS) and populationbased HIV survey. International Journal of Epidemiology, 37, 956-962. doi:10.1093/ije/dym211

[17] Rochat, T.J., et al. (2006) Depression among pregnant rural South African women undergoing HIV testing. Journal of the American Medical Association, 295, 1376-1378. doi:10.1001/jama.295.12.1376

[18] American Psychiatric Association (2000) Diagnostic and statistical manual of mental disorders (text revision). 4th Edition, American Psychiatric Association, Washington DC.

[19] Lusskin, S.I., Pundiak, T.M. and Habib, S.M. (2007) Perinatal depression: Hiding in plain sight. Canadian Journal of Psychiatry, 52, 479-488.

[20] Mann, J.J., et al. (2005) Suicide prevention strategies. Journal of the American Medical Association, 294, 2064 2074. doi:10.1001/jama.294.16.2064

[21] Taylor, S. and Jenkins, R. (2007) How are nations trying to prevent suicide? An analysis of national suicide prevention strategies. Acta Psychiatrica Scandinavica, 95, 457-463. doi:10.1111/j.1600-0447.1997.tb10132.x

[22] Schlebusch, L. (2012) Suicide prevention: A proposed national strategy for South Africa. African Journal of Psychiatry, 15, 436-440. doi:10.4314/ajpsy.v15i6.56

[23] Joe, S., et al. (2008) Non-fatal suicidal behavior among South Africans: Results from the South Africa Stress and Health Study. Social Psychiatry and Psychiatric Epidemiology, 43, 454-461. doi:10.1007/s00127-008-0348-7

[24] Burrows, S. and Laflamme, L. (2006) Suicide mortality in South Africa: A city-level comparison across socio- demographic groups. Social Psychiatry and Psychiatric Epidemiology, 41, 108-114. doi:10.1007/s00127-005-0004-4

[25] Thurlow, J., Gow, J. and George, G. (2009) HIV/AIDS, growth and poverty in KwaZulu-Natal and South Africa: An integrated survey, demographic and economy-wide 
analysis. Journal of the International AIDS Society, 12, 18. doi:10.1186/1758-2652-12-18

[26] Schlebusch, L. and Govender, R.D. (2012) Age, gender and suicidal ideation following voluntary HIV counseling and testing. International Journal of Environmental Research and Public Health, 9, 521-530. doi:10.3390/ijerph9020521

[27] Fisher, J., et al. (2012) Prevalence and determinants of common perinatal mental disorders in women in low-and lower-middle-income countries: A systematic review. Bulletin of the World Health Organization, 90, 139-149. doi:10.2471/BLT.11.091850

[28] Burrows, S. and Laflamme, L. (2005) Living circumstances of suicide mortality in a South African city: An ecological study of differences across race groups and sexes. Suicide and Life-Threatening Behavior, 35, 592-603. doi:10.1521/suli.2005.35.5.592

[29] Patel, V., et al. (2007) Prioritizing health problems in women in developing countries: Comparing the financial burden of reproductive tract infections, anaemia and depressive disorders in a community survey in India. Tropical Medicine and International Health, 12, 130-139.

[30] Patel, V., et al. (2011) Improving access to psychological treatments: Lessons from developing countries. Behaviour Research and Therapy, 49, 523-528. doi:10.1016/j.brat.2011.06.012

[31] Patel, V., et al. (2009) Packages of care for depression in low- and middle-income countries. PLoS Medicine, 6, e1000159. doi:10.1371/journal.pmed.1000159

[32] Bunevicius, R., et al. (2009) Psychosocial risk factors for depression during pregnancy. Acta Obstetricia et Gynecologica Scandinavica, 88, 599-605. doi: $10.1080 / 00016340902846049$

[33] Govender, R. and Schlebusch, L. (2012) Suicidal ideation in seropositive patients seen at a South African HIV voluntary counselling and testing clinic. African Journal of Psychiatry, 15, 94-98.

[34] Catalan, J., et al. (2011) HIV infection and mental health: Suicidal behaviour-Systematic review. Psychology, Health \& Medicine, 16, 588-611. doi:10.1080/13548506.2011.582125

[35] Carrico, A.W., et al. (2007) Correlates of suicidal ideation among HIV-positive persons. AIDS, 21, 1199-1203. doi:10.1097/QAD.0b013e3281532c96

[36] Badiee, J., et al. (2012) Lifetime suicidal ideation and attempt are common among HIV+ individuals. Journal of Affective Disorders, 136, 993-999. doi:10.1016/j.jad.2011.06.044

[37] Cooperman, N.A. and Simoni, J.M. (2005) Suicidal ideation and attempted suicide among women living with HIV/AIDS. Journal of Behavioral Medicine, 28, 149-156. doi:10.1007/s10865-005-3664-3

[38] Keiser, O., et al. (2010) Suicide in HIV-infected individuals and the general population in Switzerland, 19882008. American Journal of Psychiatry, 167, 143-150. doi:10.1176/appi.ajp.2009.09050651

[39] Carrico, A.W., Johnson, M.O., Morin, S.F., Remien, R. H., Charlebois, E.D., Steward, W.T. and Team, t. N. H. L.
P. (2007) Correlates of suicidal ideation among HIVpositive persons. AIDS, 21, 1199-1203. doi:10.1097/QAD.0b013e3281532c96

[40] Carrico, A.W. (2010) Elevated suicide rate among HIVpositive persons despite benefits of antiretroviral therapy: Implications for a stress and coping model of suicide. American Journal of Psychiatry, 167, 117-119. doi:10.1176/appi.ajp.2009.09111565

[41] Capron, D.W., et al. (2012) Suicidality and anxiety sensitivity in adults with HIV. AIDS Patient Care and STDs, 26, 298-303. doi:10.1089/apc.2011.0429

[42] Alder, J., et al. (2007) Depression and anxiety during pregnancy: A risk factor for obstetric, fetal and neonatal outcome? A critical review of the literature. Journal of Maternal and Fetal Neonatal Medicine, 20, 189-209. doi:10.1080/14767050701209560

[43] Burrows, S. and Laflamme, L. (2006) Suicide mortality in South Africa. Social Psychiatry and Psychiatric Epidemiology, 41, 108-114. doi:10.1007/s00127-005-0004-4

[44] Stein, A., et al. (2005) Babies of a pandemic. Archives of Disease in Childhood, 90, 116-118. doi:10.1136/adc.2004.049361

[45] Mkwanazi, N.B., et al. (2008) Rapid testing may not improve uptake of HIV testing and same day results in a rural South African community: A cohort study of 12,000 women. PloS One, 3, e3501. doi:10.1371/journal.pone.0003501

[46] Rochat, T.J., et al. (2011) Towards a family-centered approach to HIV treatment and care for HIV-exposed children, their mothers and their families in poorly resourced settings. Future Virology, 6, 687-696. doi: $10.2217 /$ fvl.11.45

[47] Olley, B.O., et al. (2004) Predictors of major depression in recently diagnosed patients with HIV/AIDS in South Africa. AIDS Patient Care STDS, 18, 481-487. doi:10.1089/1087291041703700

[48] Grossman, C. and Gordon, C. (2010) Mental health considerations in secondary HIV prevention. AIDS and Behavior, 14, 263-271. doi:10.1007/s10461-008-9496-8

[49] Ketchen, B., Armistead, L. and Cook, S. (2009) HIV infection, stressful life events, and intimate relationship power: The moderating role of community resources for black South African women. Women Health, 49, 197-214. doi: $10.1080 / 03630240902963648$

[50] Bilszta, J.L.C., et al. (2008) Single motherhood versus poor partner relationship: Outcomes for antenatal mental health. Australian and New Zealand Journal of Psychiatry, 42, 56-65. doi:10.1080/00048670701732731

[51] Kaaya, S.F., et al. (2010) Socio-economic and partner relationship factors associated with antenatal depressive morbidity among pregnant women in Dar es Salaam, Tanzania. Tanzanian Journal of Health Research, 12, 23-35.

[52] Lancaster, C.A., et al. (2010) Risk factors for depressive symptoms during pregnancy: a systematic review. American Journal of Obstetrics and Gynecology, 202, 5-14. doi:10.1016/j.ajog.2009.09.007

[53] Lau, Y., Yin, L. and Wang, Y. (2010) Antenatal depressive symptomatology, family conflict and social support among 
Chengdu Chinese women. Maternal and Child Health Journal, 15, 1416-1426.

[54] Pereira, M. and Canavarro, M.C. (2009) Relational contexts in adjustment to pregnancy of HIV-positive women: Relationships, social support and personal adjustment. AIDS Care, 21, 301-308. doi:10.1080/09540120802183453

[55] Callaghan, M., Ford, N. and Schneider, H. (2010) A systematic review of task-shifting for HIV treatment and care in Africa. Human Resources for Health, 8, 8.

[56] Heidari, S., Harries, A. and Zachariah, R. (2011) Facing up to programmatic challenges created by the HIV/AIDS epidemic in sub-Saharan Africa. Journal of the International AIDS Society, 14, 1-4. doi:10.1186/1758-2652-14-S1-S1

[57] Zachariah, R., et al. (2009) Task shifting in HIV/AIDS: Opportunities, challenges and proposed actions for subSaharan Africa.

[58] Petersen, I., et al. (2012) A task shifting approach to primary mental health care for adults in South Africa: Human resource requirements and costs for rural settings. Health Policy and Planning, 27, 42-51. doi:10.1093/heapol/czr012
[59] Uebel, K.E., Nash, J. and Avalos, A. (2007) Caring for the Caregivers: Models of HIV/AIDS care and treatment provision for health care workers in southern Africa. Journal of Infectious Diseases, 196, S500-S504. doi: $10.1086 / 521113$

[60] Kruse, G.R., et al. (2009) Burnout and use of HIV services among health care workers in Lusaka District, Zambia: A cross-sectional study. Human Resources for Health, 7, 55. doi:10.1186/1478-4491-7-55

[61] Paulden, M., et al. (2009) Screening for postnatal depression in primary care: Cost effectiveness analysis. British Medical Journal, 339, b5203. doi:10.1136/bmj.b5203

[62] Almond, P. and Lathlean, J. (2011) Inequity in provision of and access to health visiting postnatal depression services. Journal of Advanced Nursing, 67, 2350-2362.

[63] Collins, P.Y., et al. (2011) Grand challenges in global mental health. Nature, 475, 27-30. doi: $10.1038 / 475027 \mathrm{a}$

[64] Patel, V., et al. (2011) A renewed agenda for global mental health. The Lancet, 378, 1441-1442. doi:10.1016/S0140-6736(11)61385-8 